\title{
THE RADICAL OF THE ROW-FINITE MATRICES OVER AN ARBITRARY RING
}

\author{
BY \\ N. E. SEXAUER AND J. E. WARNOCK
}

1. Introduction. In $1956 \mathrm{~N}$. Jacobson [1, p. 23] stated the following problem. If $R$ is a ring, $J$ an infinite set and $R_{J}$ the ring of row-finite matrices with rows and columns indexed by the set $J$, determine the radical of $R_{J}$. All rings considered here are associative and by the radical is meant the Jacobson radical. In 1962 E. M. Patterson [3] contributed to the solution of this problem when he proved that the radical of $R_{\mathrm{J}}$ coincides with the set of row-finite matrices over the radical of $R$ if and only if the radical of $R$ is right vanishing. By extending the concept of a right vanishing set of a ring to that of a right vanishing family of left ideals in a ring, we obtain the following solution to the problem. If $A$ is in $R_{J}$ and $\mathfrak{A}_{\lambda}$ is the left ideal of $R$ generated by the elements of the $\lambda$ th column of $A$, then $A$ is in the radical of $R_{J}$ if and only if each element of $A$ is in the radical of $R$ and the totality of the $\mathfrak{A}_{\lambda}$, as $\lambda$ ranges over the columns of $A$, is a right vanishing family of left ideals in $R$.

In order to obtain this theorem it will be necessary to show the validity of solutions of certain types of equations over an arbitrary ring and to prove three additional results, This will be done in the next three sections.

2. The equations. Since we must differentiate between the two types of quasiregularity of elements in a ring, we recall these definitions and some basic properties. Let $R$ be a ring and $a$ an element of $R$. $a$ is quasi-regular if there exists an element $a^{\prime}$ in $R$ such that $a+a^{\prime}-a a^{\prime}=0=a+a^{\prime}-a^{\prime} a, a^{\prime}$ is unique and is the quasi-inverse of $a$. $a$ is plus quasi-regular if there exists an element $a^{\prime \prime}$ in $R$ such that $a+a^{\prime \prime}+a a^{\prime \prime}$ $=0=a+a^{\prime \prime}+a^{\prime \prime} a, a^{\prime \prime}$ is unique and is the plus quasi-inverse of $a$. We shall consistently use throughout this paper the notation $a^{\prime}$ and $a^{\prime \prime}$ for the quasi-inverse and plus quasi-inverse of $a$ respectively. It is well known that $a$ is quasi-regular if and only if $R(1-a)=R=(1-a) R$, while $a$ is plus quasi-regular if and only if $R(1+a)=R=(1+a) R$, where $R(1-a)=\{x-x a \mid x \in R\}$. From these characterizations it is easy to see that every quasi-regular one-sided ideal in $R$ has the property that each of its elements is plus quasi-regular. Similarly every plus quasi-regular one-sided ideal has the property that each of its elements is quasi-regular.

Proposition 1. If $a$ is a quasi-regular element in $R$ and $b$ is in $R$, then there exists $a$ unique element $x$ in $R$ such that $x-x a=b$. Moreover $x=b-b a^{\prime}$.

Received by the editors August 3, 1964 and, in revised form, October 10, 1967. 
Proof. Existence is given by $R(1-a)=R$. If $x-x a=b$, then since $a+a^{\prime}-a a^{\prime}=0$, $0=x a+(x-x a) a^{\prime}=x a+b a^{\prime}$. Therefore $x=b+x a=b-b a^{\prime}$.

It can also be seen that the existence of unique solutions of the equations $x-a x=b, x+x a=b$ and $x+a x=b$ is assured if $a$ is quasi-regular in the first case and $a$ is plus quasi-regular in the last two cases. The solutions are $b-a^{\prime} b, b+b a^{\prime \prime}$ and $b+a^{\prime \prime} b$ respectively.

If $R$ is a ring, let $\Gamma(R)$ denote the radical of $R . \Gamma(R)$ is a quasi-regular ideal which contains every quasi-regular one-sided ideal [1].

Proposition 2. If $a$ is in $\Gamma(R)$ and $b$ is in $R$, then there exists a unique element $x$ in $R$ such that $x-(x a)^{\prime} x=b$. Moreover $x=b+b(a b)^{\prime \prime}$.

Proof. Since $b+b\left((a b)^{\prime \prime}+a b+(a b)^{\prime \prime} a b\right)=b, b+b(a b)^{\prime \prime}=b-\left(b+b(a b)^{\prime \prime}\right) a b$. If $x=$ $b+b(a b)^{\prime \prime}$, then

$$
\begin{aligned}
x-(x a)^{\prime} x & =b-\left(b+b(a b)^{\prime \prime}\right) a b-\left(\left(b+b(a b)^{\prime \prime}\right) a\right)^{\prime}\left(b-\left(b+b(a b)^{\prime \prime}\right) a b\right) \\
& =b-\left[b a+b(a b)^{\prime \prime} a+\left(b a+b(a b)^{\prime \prime} a\right)^{\prime}-\left(b a+b(a b)^{\prime \prime} a\right)^{\prime}\left(b a+b(a b)^{\prime \prime} a\right)\right] b \\
& =b .
\end{aligned}
$$

To show uniqueness, let $x$ be an element of $R$ such that $x-(x a)^{\prime} x=b$. If $c=(x a)^{\prime}$, then $x-c x=b$ and $c$ is quasi-regular. Hence $x=b-c^{\prime} b=b-x(a b)$ and hence $x+x(a b)=b$. It follows that $x=b+b(a b)^{\prime \prime}$ since $a b$ is plus quasi-regular.

Similarly we can show the existence of unique solutions of the equations $x-x(a x)^{\prime}=b, x+x(a x)^{\prime \prime}=b$ and $x+(x a)^{\prime \prime} x=b$ if $a$ is in $\Gamma(R)$. The solutions are $b+(b a)^{\prime \prime} b, b-(b a)^{\prime} b$ and $b-b(a b)^{\prime}$ respectively.

3. Right vanishing families of left ideals. Let $R$ be a ring, $J$ an infinite index set and $\left\{\mathfrak{A}_{\lambda} \mid \lambda \in J\right\}$ a family of subsets of $R$. $\left\{\mathfrak{A}_{\lambda} \mid \lambda \in J\right\}$ will be called a right vanishing family of subsets of $R$ if for every sequence $\left\{\mathfrak{A}_{\lambda_{n}} \mid n=1,2, \ldots\right\}$ of subsets in $\left\{\mathfrak{U}_{\lambda} \mid \lambda \in J\right\}$ such that $\lambda_{n} \neq \lambda_{m}$ if $n \neq m$, and every sequence of elements $\left\{a_{n} \mid n=1,2, \ldots\right\}$ where $a_{n} \in \mathfrak{A}_{\lambda_{n}}$ for $n=1,2, \ldots$, there exists a positive integer $r$, depending on $\left\{a_{n} \mid n=1,2, \ldots\right\}$ such that the product $a_{1} a_{2} \cdots a_{r}=0$.

Proposition 3. Let $\left\{S_{\lambda} \mid \lambda \in J\right\}$ be a family of subsets of a ring $R$. For each $\lambda$ in $J$, let $\mathfrak{N}_{\lambda}^{*}$ be the left ideal in $R$ consisting of the finite sums $\sum x_{i} a_{i}$ where $x_{i}$ is in $R$ and $a_{i}$ is in $S_{\lambda}$, and let $\mathfrak{A}_{\lambda}$ be the left ideal in $R$ generated by the set $S_{\lambda}$. Then $\left\{\mathfrak{U}_{\lambda}^{*} \mid \lambda \in J\right\}$ is a right vanishing family of left ideals if and only if $\left\{\mathfrak{U}_{\lambda} \mid \lambda \in J\right\}$ is a right vanishing family of left ideals.

Proof. Since $\mathfrak{Q}_{\lambda}^{*} \leqq \mathfrak{A}_{\lambda}$ for all $\lambda$ in $J$, if $\left\{\mathfrak{A}_{\lambda} \mid \lambda \in J\right\}$ is a right vanishing family of left ideals, then so is $\left\{\mathfrak{U}_{\lambda}^{*} \mid \lambda \in J\right\}$. Next assume by way of contradiction that there exists a sequence $\left\{\mathfrak{A}_{\lambda_{n}} \mid n=1,2, \ldots\right\}$ of left ideals in $\left\{\mathfrak{A}_{\lambda} \mid \lambda \in J\right\}$ such that $\lambda_{n} \neq \lambda_{m}$ if $n \neq m$ and a sequence $\left\{a_{n} \in \mathfrak{A}_{\lambda_{n}} \mid n=1,2, \ldots\right\}$ such that for every positive integer 
$r, a_{1} a_{2} \cdots a_{r} \neq 0$. Let $c_{n}=a_{2 n-1} a_{2 n}$, then $c_{1} c_{2} \cdots c_{r}=a_{1} a_{2} \cdots a_{2 r} \neq 0$ for every $r$ and $c_{n}$ is an element of $\mathfrak{A}_{\lambda_{2 n}}^{*}$. Using the sequence $\left\{\mathfrak{U}_{\lambda_{2 n}}^{*} \mid n=1,2, \ldots\right\}$ we obtain a contradiction of the assumption that $\left\{\mathfrak{A}_{\lambda}^{*} \mid \lambda \in J\right\}$ is a right vanishing family of left ideals.

4. Two lemmas. In the remainder of this paper $R$ will be a ring, $J$ an infinite index set and $R_{J}$ the ring of $J \times J$ row-finite matrices over $R$. The following notation will be used. As above $\Gamma(R), \Gamma\left(R_{J}\right)$ will be the radicals of $R$ and $R_{J}$ respectively and $(\Gamma(R))_{J}$ will denote the set of $J \times J$ row-finite matrices over $\Gamma(R)$. If $B$ is an element of $R_{J}$ and $S_{1}$ and $S_{2}$ are subsets of $J$, then $B_{S_{1} \times S_{2}}$ will denote the restriction of $B$ to $S_{1} \times S_{2}$. If $S_{2}$ consists of the single element $\lambda$, we shall write $B_{S_{1} \times \lambda}$ for $B_{S_{1} \times S_{2}}$. We will let $\{B\}_{S_{1} \times S_{2}}$ denote the set of elements in the matrix $B_{S_{1} \times S_{2}}$ and in general if $M$ is a given matrix, then $\{M\}$ will represent the set of elements in $M$. If $A=\left|a_{\lambda \mu}\right|$ is in $R_{J}$ then for each $\lambda$ in $J$ let $\mathfrak{A}_{\lambda}$ denote the left ideal in $R$ generated by $\{A\}_{J \times \lambda}$ and let $\mathfrak{A}_{\lambda}^{*}$ denote the left ideal in $R$ consisting of the finite sums $\sum x_{i} a_{i \lambda}$ where $x_{i}$ is in $R$ and $a_{i \lambda}$ is in $\{A\}_{J \times \lambda}$.

In Lemmas 1 and 2 and in the necessity half of the proof of the theorem we will also use the following notation. Well order $J$ and let this ordering be denoted by $<$. We will say that a matrix $C=\left|c_{\lambda \mu}\right|$ in $R_{J}$ is upper triangular if $c_{\lambda \mu}=0$ for $\mu \leqq \lambda$.

LEMMA 1. If $C=\left|c_{\lambda \mu}\right|$ is an upper triangular matrix in $R_{J}$ and if $\left\{\bigodot_{\lambda} \mid \lambda \in J\right\}$ is a right vanishing family of left ideals of $R$, then $C$ is quasi-regular.

Proof. Denote the elements of the $n$th power of $C$ by $c_{\lambda \mu}^{n}$. For each $\lambda$ in $J$ define recursively a sequence of finite subsets of $J$ as follows. Let $S_{1}$ consist of those $\phi$ in $J$ for which $c_{\lambda \phi} \neq 0$, and $S_{n+1}$ consist of those $\phi$ in $J$ for which there exists a $\mu$ in $S_{n}$ such that $c_{\mu \phi} \neq 0$. We then have for each $n \geqq 2$ and for each $\mu$ in $J$ that

$$
c_{\lambda \mu}^{n}=\sum c_{\lambda \phi_{1}} c_{\phi_{1} \phi_{2}} \cdots c_{\phi_{n-1} \mu}
$$

where in the sum the range is given by $\phi_{1} \in S_{1}, \phi_{2} \in S_{2}, \ldots, \phi_{n-1} \in S_{n-1}$.

We will first show that for each $\lambda$ in $J$ there exists a positive integer $n(\lambda)$ such that for all $n>n(\lambda), c_{\lambda \mu}^{n}=0$ for all $\mu$ in $J$. To this end, we note that if given a $\lambda$, a corresponding $S_{r}=\varnothing$, then $n(\lambda)=r$ has this property. Thus we may assume that each $S_{n} \neq \varnothing$. Next assume by way of contradiction that there exists a $\lambda$ in $J$ with the property that for each positive integer $n$ there exists a positive integer $m>n$ such that $c_{\lambda \mu}^{m} \neq 0$ for all $\mu$ in $J$. From this it follows that there exists a sequence of positive integers $M=\left\{m_{n} \mid n=1,2, \ldots\right\}$ and a sequence $U=\left\{\mu_{n} \mid n=1,2, \ldots\right\}$ of elements of $J$ such that $1<m_{1}, m_{n}<m_{n+1}$ and $c_{\lambda \mu_{n}}^{m_{n}} \neq 0$ for all $n$. Since $c_{\lambda \mu_{n}}^{m_{n}} \neq 0$ we see from (i) that for each $n=2,3, \ldots$ there exists indices $\phi_{j}(n)\left(j=1,2, \ldots, m_{n}-1\right)$ such that $c_{\lambda \phi_{1}(n)} c_{\phi_{1}(n) \phi_{2}(n)} \cdots c_{\phi_{m_{n}-1}(n) \mu_{n}} \neq 0$ and that each of these summands have at least three factors, since $1<m_{1}<m_{2}<\cdots$ and since the terms in (i) for $c_{\lambda \mu}^{n}$ have $n$ factors. Let $T_{1}$ consist of the $\phi_{1}(n)$ for $n=2,3, \ldots$ Since $T_{1} \leqq S_{1}$ and since $S_{1}$ is finite we see that there exists a $\lambda_{1}$ in $J$ and an infinite subset $P_{1} \leqq M$ such that for 
all $m_{n}$ in $P_{1}$ we have that $\phi_{1}(n)=\lambda_{1}$ and that for the corresponding $\mu_{n}$ in $U$ we have that $c_{\lambda \lambda_{1}} c_{\lambda_{1} \phi_{2}(n)} \cdots c_{\phi_{m_{n}-1}(n) \mu_{n}}$ is a nonzero summand occurring in the formula (i) for $c_{\lambda \mu_{n}}^{m_{n}}$. Moreover since $c_{\lambda \lambda_{1}} \neq 0, \lambda<\lambda_{1}$ since $C$ is upper triangular. Thus, using induction, we can construct a sequence $\left\{\lambda_{n} \mid n=1,2, \ldots\right\}$ of elements of $J$ with the properties that $\lambda<\lambda_{1}, \lambda_{n}<\lambda_{n+1}$ for all $n$ and such that for each positive integer $r$ there exists $m_{n}$ in $M$ and corresponding $\mu_{n}$ in $U$ with $m_{n}>r+1$ and there exists indices $\phi_{j}(n)\left(j=1,2, \ldots, m_{n}-1\right)$ such that

$$
c_{\lambda \lambda_{1}} c_{\lambda_{1} \lambda_{2}} \cdots c_{\lambda_{r-1} \lambda_{r}} c_{\lambda_{r} \phi_{r+1}(n)} \cdots c_{\phi_{m_{n}-1}(n) \mu_{n}}
$$

is a nonzero summand occurring in the formula (i) for $c_{\lambda \mu_{n}}^{m_{n}}$, hence $c_{\lambda \lambda_{1}} c_{\lambda_{1} \lambda_{2}} \cdots c_{\lambda_{r-1} \lambda_{r}}$ $\neq 0$. For this sequence we see that $c_{\lambda_{1}} \in \mathbb{E}_{\lambda_{1}}$ and $c_{\lambda_{n} \lambda_{n+1}} \in \mathfrak{E}_{\lambda_{n+1}}$ for $n=1,2, \ldots$, and that since $\lambda_{n}<\lambda_{n+1}$ for all $n, \lambda_{n} \neq \lambda_{m}$ if $n \neq m$. It follows, since $\left\{\mathfrak{E}_{\lambda} \mid \lambda \in J\right\}$ is a right vanishing family of left ideals, that there exists a positive integer $r$ such that $c_{\lambda \lambda_{1}} c_{\lambda_{1} \lambda_{2}} \cdots c_{\lambda_{r-1} \lambda_{r}}=0$. This contradicts a property of the constructed sequence.

Next define a matrix $\bar{C}=\left|\bar{c}_{\lambda \mu}\right|$ as follows. Given $\lambda$ in $J$ let $n(\lambda)$ be as just found and define for each $\mu$ in $J \bar{c}_{\lambda \mu}=c_{\lambda \mu}+c_{\lambda \mu}^{2}+\cdots+c_{\lambda \mu}^{n(\lambda)}$. Since $C, C^{2}, \ldots, C^{n(\lambda)}$ are all row-finite, $\bar{C}$ is in $R_{\mathrm{J}}$. Let $C^{\prime}=-\bar{C}$. We first show that $C^{\prime}$ is a left quasi-inverse of $C$. Thus take $\lambda$ in $J$ and let $e(\mu)$ be the $(\lambda, \mu)$ th element of $C-\bar{C}+\bar{C} C$. Let $S$ be the finite subset of $J$ consisting of all $\phi$ such that $\bar{c}_{\lambda \phi} \neq 0$, and let $n(\lambda)$ be the positive integer as found above. For $1 \leqq k \leqq n(\lambda)$ let $T_{k}$ be a finite subset of $J$ such that $c_{\lambda \phi}^{k}=0$ if $\phi \notin T_{k}$, and let $T$ be the union of $T_{1}, \ldots, T_{n(\lambda)}$. It follows that $S \leqq T$ and that $c_{\lambda \mu}^{k}=\sum_{\phi \in T} c_{\lambda \phi}^{k-1} c_{\phi \mu}$ for all $\mu$ in $J(k=2, \ldots, n(\lambda)+1)$. Then using $c_{\lambda \mu}^{n(\lambda)+1}=0$ and $\bar{c}_{\lambda \phi}=c_{\lambda \phi}+\cdots+c_{\lambda \phi}^{n(\lambda)}$ we have

$$
e(\mu)=-\left(c_{\lambda \mu}^{2}+\cdots+c_{\lambda \mu}^{n(\lambda)+1}\right)+\sum_{\phi \in S} \bar{c}_{\lambda \phi} c_{\phi \mu}=-\left(\sum_{\phi \in T-S}\left(\sum_{n=1}^{n(\lambda)} c_{\lambda \phi}^{n}\right) c_{\phi \mu}\right) .
$$

But if $\phi$ is in $T-S$, then $\bar{c}_{\lambda \phi}=0$, hence $\sum_{n=1}^{n(\lambda)} c_{\lambda \phi}^{n}=0$, thus $e(\mu)=0$. To show that $C^{\prime}$ is a right quasi-inverse of $C$, take $\lambda$ in $J$ and let $f(\mu)$ be the $(\lambda, \mu)$ th element of $C-\bar{C}+C \bar{C}$. Let $V$ be the finite subset of $J$ consisting of all $\phi$ such that $c_{\lambda \phi} \neq 0$. Further let $n(\lambda)$ and $n(\phi)$, for each $\phi$ in $V$, be as found in the last paragraph. Let $n_{0}$ be the maximum of $n(\lambda)$ and the $n(\phi)$ for $\phi$ in $V$. Then for each $\mu$ in $J, \bar{c}_{\lambda \mu}=c_{\lambda \mu}+$ $\cdots+c_{\lambda \mu}^{n_{0}}$ and $\bar{c}_{\phi \mu}=c_{\phi \mu}+\cdots+c_{\phi \mu}^{n_{0}}$ for each $\phi$ in $V$. We also have that

$$
c_{\lambda \mu}^{k}=\sum_{\phi \in V} c_{\lambda \phi} c_{\phi \mu}^{k-1} \quad \text { for } k=2, \ldots, n_{0}+1
$$

It follows that $f(\mu)=0$.

For the next lemma let 1 denote the first element of $J$ and $n$ the $n$th element in the well ordering of $J, I$ the subset of elements of $J$ with only a finite number of predecessors and $I^{\prime}$ the complement of $I$ in $J$.

Lemma 2. Let $A=\left|a_{i j}\right|$ be in $R_{\mathrm{J}}$. If $\left\{\mathfrak{H}_{\lambda_{m}}^{*} \mid m \in I\right\}$ is a sequence of left ideals in $\left\{\mathfrak{A}_{\lambda}^{*} \mid \lambda \in J\right\}$ such that $\lambda_{m} \neq \lambda_{n}$ if $m \neq n$, and if $\left\{c_{m} \mid m \in I\right\}$ is a sequence of elements of $R$ such that $c_{m} \in \mathfrak{A}_{\lambda_{m}}^{*}$ for $m$ in $I$, then there exists a subsequence $\left\{c_{m_{n}} \mid n \in I\right\}$ of 
$\left\{c_{m} \mid m \in I\right\}$ such that $m_{1}=2$ and $m_{n}+2<m_{n+1}$ for $n$ in $I$, and there exists a matrix $F=\left|f_{i j}\right|$ in the ideal generated by $A$ in $R_{J}$ which has the following properties:

(1) $f_{i 1}=0$ for $i$ in $J$.

(2) $f_{n, n+1}=c_{m_{n}} c_{m_{n}+1}$ for $n$ in 1 .

(3) If $n$ is in $I, j$ in $J$ and $n+1<j$, then $f_{n j}=0$.

(4) If $\mu$ is in $I^{\prime}$, then $f_{\mu j}=0$ for $j$ in $J$.

Proof. We first define the subsequence and simultaneously construct a matrix $B=\left|b_{i j}\right|$ in $R_{J}$ with the property: If $D=B A$, then (i) the $\left(n, \lambda_{m_{n}}\right)$ th element of $D$ is $c_{m_{n}}$ for $n$ in $I$, (ii) if $1 \leqq k<n$, then the $\left(k, \lambda_{m_{n}}\right)$ th element of $D$ is 0 for $k, n$ in $I$, (iii) if $\mu$ is in $I^{\prime}$, then the $(\mu, j)$ th element of $D$ is 0 for $j$ in $J$. To this end, if $\mu$ is in $I^{\prime}$, define $b_{\mu j}=0$ for all $j$ in $J$, hence (iii) holds. We now define inductively the remaining rows of $B$ and the subsequence. Since $c_{2} \in \mathfrak{H}_{\lambda_{2}}^{*}$, there exists a finite subset $S_{1}$ of $J$ and a set $T_{1}=\left\{t_{1 i} \mid i \in S_{1}\right\}$ of elements of $R$ such that $\sum_{i \in S_{1}} t_{1 i} a_{i \lambda_{2}}=c_{2}$. Define the first row of $B$ as follows. Let $b_{1 j}=t_{1 j}$ if $j \in S_{1}$ and $b_{1 j}=0$ if $j \notin S_{1}$. Define $m_{1}=2$. Then (i) and (ii) hold for $n=1$. Assume that the first $p$ rows of $B$ have been defined and that $c_{m_{1}}, \ldots, c_{m_{p}}$ have been defined such that $m_{n}+2<m_{n+1}$ and (i) and (ii) hold for $n=1,2, \ldots, p$. There exists finite subsets $S_{1}, S_{2}, \ldots, S_{p}$ in $J$ such that if $1 \leqq n \leqq p$ and $j \notin S_{n}$, then $b_{n j}=0$ for $j \in J$. Let $M$ be the union of $S_{1}, \ldots, S_{p}$. If $i$ is in $M$, then there exists a finite subset $S_{i}^{\prime}$ of $J$ such that if $j \notin S_{i}^{\prime}, a_{i j}=0$. Let $M^{\prime}$ be the union of the $S_{i}^{\prime}$ as $i$ ranges over the finite set $M$. Using the assumption given on $\left\{\mathfrak{U}_{\lambda_{m}}^{*} \mid m \in I\right\}$, and the fact that $M^{\prime}$ is finite we see that there exists a $q$ in $I$ such that $m_{p}+2<q$ and $\lambda_{q} \notin M^{\prime}$. Pick such a $q$ and define $m_{p+1}=q$. Since $c_{q} \in \mathfrak{A}_{\lambda_{q}}^{*}$, there exists a finite subset $S_{p+1}$ of $J$ and a set $T_{p+1}=\left\{t_{p+1, i} \mid i \in S_{p+1}\right\}$ of elements in $R$ such that $\sum_{i \in S_{p+1}} t_{p+1, i} a_{i \lambda_{q}}=c_{q}$. Define the $(p+1)$ th row of $B$ as follows. Let $b_{p+1, j}=t_{p+1, j}$ if $j \in S_{p+1}$ and $b_{p+1, j}=0$ if $j \notin S_{p+1}$. Then (i) and (ii) hold for $n=p+1$. That (ii) holds follows from the fact that $\lambda_{q} \notin M^{\prime}$ implies that $\lambda_{q} \notin S_{i}^{\prime}$ for $i$ in $M^{\prime}$.

Next define a matrix $E=\left|e_{i j}\right|$ in $R_{J}$ as follows. Let $e_{i 1}=0$ for all $i$ in $J$. If $n \in I$, $i$ in $J$ and $i \neq \lambda_{m_{n}}$, let $e_{i, n+1}=0$. If $n$ is in $I$ and $i=\lambda_{m_{n}}$, let $e_{i, n+1}=c_{m_{n}+1}$. If $\mu$ is in $I^{\prime}$, let $e_{i \mu}=0$ for all $i$ in $J$.

Let $F=D E$. Then $F$ is in $R_{J}$ and satisfies the conclusions of the lemma.

\section{The theorem.}

THEOREM. Let $A$ be an element of $R_{J}$. Then necessary and sufficient conditions that $A$ is in $\Gamma\left(R_{J}\right)$ are that $A$ is in $(\Gamma(R))_{J}$ and that $\left\{\mathfrak{A}_{\lambda} \mid \lambda \in J\right\}$ is a right vanishing family of left ideals of $R$.

Proof. We first show the sufficiency of the two conditions.

Sufficiency. That $A$ is in $\Gamma\left(R_{J}\right)$ will follow if we show that the principal left ideal in $R_{J}$ generated by $A$ is quasi-regular. Assume then that $B=\left|b_{i j}\right|$ is an element in the left ideal generated by $A$. We will construct a left quasi-inverse $B^{\prime}$ in $R_{J}$ of $B$. This will be done by constructing an arbitrary row of $B^{\prime}$. To this end let $p$ be an element of $J$. We will have need of the following finite sets, defined recursively, 
which are associated with the elements of the $p$ th row of the powers of $B$. Let $S_{1}$ consist of $p$ and those $j$ in $J$ for which $b_{p j} \neq 0$. Let $S_{n+1}$ consist of those $j$ in $J$ for which $j$ is not in the union of $S_{1}, \ldots, S_{n}$ and for which there exists an $i$ in $S_{n}$ such that $b_{i j} \neq 0$. Also let $S(n)$ denote the union of the sets $S_{1}, \ldots, S_{n}$. It is seen that the intersection of $S_{n}$ and $S_{m}$ is null if $n \neq m$ and that if $m+1<k$ and $S_{k} \neq \varnothing$, then $B_{S_{m} \times S_{k}}=|0|$. The last result follows if we note that necessarily $S_{m} \neq \varnothing$ and that if $i$ is in $S_{m}$ and $b_{i j} \neq 0$, then $j$ is in the union of $S_{1}, \ldots, S_{m}$ or $j$ is in $S_{m+1}$.

Let $I_{p}$ denote the union of the sets $S_{n}$ for $n=1,2, \ldots$ We see that $I_{p}$ is either a nonempty finite set or is countably infinite. Well order $I_{p}$ in the following manner. Let the first element be $p$, then choose the remaining elements of $S_{1}$ in some manner and let these elements be the second, third,... elements of $I_{p}$ in the well ordering. Then exhaust $S_{2}$ to determine the next finite number of elements in the well ordering. Continue in this way with $S_{3}, S_{4}, \ldots$ We now prove that there exists a matrix $\bar{B}=\left|\bar{b}_{i j}\right|$ in $(\Gamma(R))_{I_{p}}$ such that $C=\bar{B} \circ B_{I_{p} \times I_{p}}$ is an upper triangular, relative to the above well ordering of $I_{p}$, matrix in $R_{I_{p}}$ with the property that $c_{i j} \in \mathfrak{B}_{j}$ for all $i, j$ in $I_{p}$. Here $C=\left|c_{i j}\right|, \mathfrak{B}_{j}$ is the left ideal in $R$ generated by $\{B\}_{J \times j}$ and $x \circ y$ $=x+y-x y$. To see this, for each positive integer $n$, let $B_{n}=B_{S(n) \times S(n)}$. Since $\{B\} \leqq \Gamma(R)$, for $\{A\} \leqq \Gamma(R)$, and since $\Gamma\left(R_{S(n)}\right)=(\Gamma(R))_{S(n)}$, [1, p. 11], the finite matrix $B_{n}$ has a quasi-inverse $B_{n}^{\prime}=\left|\tilde{b}_{i j}\right|$ in $(\Gamma(R))_{S(n)}$. We now use this sequence of matrices $\left\{B_{n}^{\prime} \mid n=1,2, \ldots\right\}$ to define an arbitrary row of $\bar{B}$. Thus take $i$ in $I_{p}$, there exists a unique $n$ such that $i$ is in $S_{n}$. Define $\bar{b}_{i j}=\tilde{b}_{i j}$ for $j$ in $S(n)$ and $\bar{b}_{i j}=0$ for $j$ in $I_{p}$ and $j$ not in $S(n)$. Since $S(n)$ is finite $\bar{B}$ is row-finite, moreover $\bar{B}$ is in $(\Gamma(R))_{I_{p}}$. We show that $C$ is upper triangular and that $c_{i j} \in \mathfrak{B}_{j}$ by computing the elements of an arbitrary row of $C$. Take $i$ in $I_{p}$ and let $n$ be the associated positive integer given in the definition of $\bar{B}$. If $j$ is in $S(n)$, then $c_{i j}=0$ since $B_{n}^{\prime} \circ B_{n}=0$. Next if $j$ is in $S_{k}$ for $k \geqq n+2$, then $b_{i j}=0=\bar{b}_{i j}$ and if $\phi$ is in $S(n)$ then $b_{\phi j}=0$, thus $c_{i j}=0$. Finally if $j$ is in $S_{n+1}$ then $\bar{b}_{i j}=0$ and therefore $c_{i j}$ is in $\mathfrak{B}_{j}$.

We next note that if $I_{p}$ is infinite then $\left\{\mho_{j} \mid j \in I_{p}\right\}$ is a right vanishing family of left ideals of $R$. This follows from $c_{i j} \in \mathfrak{B}_{j}$ for all $i, j$ in $I_{p}$ and the fact that since $\left\{\mathfrak{U}_{\lambda} \mid \lambda \in J\right\}$ is a right vanishing family of left ideals then so is $\left\{\mathfrak{B}_{\lambda} \mid \lambda \in J\right\}$ since $\mathfrak{B}_{\lambda} \leqq \mathfrak{U}_{\lambda}$ for all $\lambda$ in $J$. Thus in this case, since $C$ is an upper triangular matrix in $R_{I_{p}}$ it follows by Lemma 1 that $C$ is a quasi-regular element in $R_{I_{p}}$. On the other hand if $I_{p}$ is finite, $C$ is nilpotent since it is upper triangular, hence is quasi-regular. Therefore in either case there is a $C^{\prime}$ in $R_{I_{p}}$ such that $C^{\prime} \circ C=0$. Let $D=C^{\prime} \circ \bar{B}$, then $D=\left|d_{i j}\right|$ is in $R_{I_{p}}$ and $D \circ B_{I_{p} \times I_{p}}=0$. We now define the $p$ th row of $B^{\prime}=\left|b_{i j}^{*}\right|$. If $j$ is in $I_{p}$, let $b_{p j}^{*}=d_{p j}$, and if $j$ is not in $I_{p}$, let $b_{p j}^{*}=0$. Since $D$ is row-finite, this row of $B^{\prime}$ has only a finite number of nonzero elements.

There remains to show that $B^{\prime}$ is a left quasi-inverse of $B$. This will follow if we show that if $p$ is in $J$, then $b_{p j}+b_{p j}^{*}-\sum_{i \in J} b_{p i}^{*} b_{i j}=0$ for all $j$ in $J$. Thus choose $p$ in $J$ and consider the sets $S_{n}$ and $I_{p}$ as defined previously.

Case 1. If $j$ is in $I_{p}$, then we must show $b_{p j}+b_{p j}^{*}-\sum_{i \in I_{p}} b_{p i}^{*} b_{i j}=0$, since $b_{p i}^{*}=0$ if $i$ is not in $I_{p}$. This last equation is valid since $D \circ B_{I_{p} \times I_{p}}=0$. 
Case 2. If $j$ is not in $I_{p}$, then $b_{p j}^{*}=0=b_{p j}$. Further $b_{p i}^{*}=0$ if $i$ is not in $I_{p}$. Hence in this case we must show that $-\sum_{i \in I_{p}} b_{p i}^{*} b_{i j}=0$. This follows if we show that $b_{i j}=0$ for all $i$ in $I_{p}$. But if $i$ is in $I_{p}$, then $i$ is in $S_{k}$ for some $k \geqq 1$. Moreover $j$ is not in the union of $S_{1}, \ldots, S_{k}$, hence if $b_{i j} \neq 0$, then $j$ is in $S_{k+1}$ and therefore in $I_{p}$. This proves the sufficiency.

Necessity. That $A$ is in $(\Gamma(R))_{J}$ follows from the relation $\Gamma\left(R_{J}\right) \leqq(\Gamma(R))_{J}$ proved by Patterson [2]. Assume by way of contradiction that $\left\{\mathfrak{A}_{\lambda} \mid \lambda \in J\right\}$ is not a right vanishing family of left ideals of $R$. It follows, by Proposition 3, that $\left\{\mathfrak{H}_{\lambda}^{*} \mid \lambda \in J\right\}$ is not a right vanishing family of left ideals of $R$. Hence there exists a sequence $\left\{\mathfrak{U}_{\lambda_{m}}^{*} \mid m \in I\right\}$ of $\left\{\mathfrak{U}_{\lambda}^{*} \mid \lambda \in J\right\}$ such that $\lambda_{m} \neq \lambda_{n}$ if $m \neq n$ and a sequence $\left\{c_{m} \mid m \in I\right\}$ of elements in $R$ such that $c_{m} \in \mathfrak{A}_{\lambda_{m}}^{*}$ for $m$ in $I$ and the product $c_{1} c_{2} \cdots c_{n} \neq 0$ for all $n$ in $I$.

Upon applying Lemma 2 to $A$, we conclude that there exists a subsequence $\left\{c_{m_{n}} \mid n \in I\right\}$ of $\left\{c_{m} \mid m \in I\right\}$ and a matrix $F=\left|f_{i j}\right|$ in the ideal generated by $A$ in $R_{J}$ which satisfies the conclusions of the lemma. Before proceeding we introduce the following notation. Let $k_{1}=c_{1}$. For $n$ in $I$ let $k_{n+1}=c_{m_{n}} c_{m_{n}+1}$ and $k_{n+1}^{*}$ $=c_{m_{n}+2} c_{m_{n}+3} \cdots c_{m_{n+1}-1}$. Hence $k_{1} k_{2} k_{2}^{*} k_{3} k_{3}^{*} \cdots k_{n} k_{n}^{*} k_{n+1}=c_{1} c_{2} \cdots c_{m_{n}+1} \neq 0$ for every $n$ in $I$.

Since $A$ is in $\Gamma\left(R_{J}\right)$ and $F$ is in the ideal generated by $A$, it follows that $F$ is in $\Gamma\left(R_{J}\right)$. Using this last result and the relation $\Gamma\left(R_{J}\right) \leqq(\Gamma(R))_{J}$, we see that the elements of $F$ are in $\Gamma(R)$. Hence, we may define, using Proposition 2, a sequence $\left\{x_{n} \mid n \in I\right\}$ of elements of $R$ as follows. Let $x_{1}=k_{1}$. Let $x_{2}$ be the element in $R$ such that $x_{2}-\left(x_{2} f_{22}\right)^{\prime} x_{2}=k_{2}^{*}$. And in general let $x_{n}$ be the element in $R$ such that

$$
\begin{aligned}
x_{n}-\left(x _ { n } \left(f_{n n}+f_{n n-1} k_{n-1}^{*} k_{n}+f_{n n-2} k_{n-2}^{*} k_{n-1} k_{n-1}^{*} k_{n}+\cdots\right.\right. & \\
& \left.\left.+f_{n 2} k_{2}^{*} k_{3} k_{3}^{*} \cdots k_{n-1} k_{n-1}^{*} k_{n}\right)\right)^{\prime} x_{n}=k_{n}^{*} .
\end{aligned}
$$

Next define a matrix $G=\left|g_{i j}\right|$ in $R_{J}$ as follows. If $i, j$ are in $J$ and $i \neq j$, let $g_{i j}=0$. Let $g_{n n}=x_{n}$ for $n$ in $I$ and $g_{i i}=0$ for $i$ in $I^{\prime}$. Let $H=G F$, then since $F \in \Gamma\left(R_{J}\right)$, $H \in \Gamma\left(R_{J}\right)$ and therefore $H$ has a quasi-inverse $H^{\prime}$. Hence we have

(i) $H+H^{\prime}-H^{\prime} H=|0|$,

(ii) $H+H^{\prime}-H H^{\prime}=|0|$.

Denote the elements of $H$ by $h_{i j}$ and those of $H^{\prime}$ by $h_{i j}^{*}$. By (3) and (4) of Lemma 2 we see that $f_{j \mu}=0$ for all $j$ in $J$ when $\mu$ is in $I^{\prime}$. This result coupled with the fact that $g_{\mu j}=0$ for $j$ in $J$ implies that if $\mu$ is in $I^{\prime}$, then $h_{\mu j}=h_{j \mu}=0$ for $j$ in $J$. Then $h_{\mu j}=0$ and (ii) imply that $h_{\mu j}^{*}=0$, while $h_{j \mu}=0$ and (i) imply that $h_{j \mu}^{*}=0$ for $j$ in $J$ and $\mu$ in $I^{\prime}$. Using this last result and noticing that $h_{12}=k_{1} k_{2} \neq 0$ implies that the elements of the first row of $H^{\prime}$ are not all zero (look at $h_{12}^{*}$ given by (i)), we see that nonzero elements in the first row of $H^{\prime}$ exist and that they occur among the set $\left\{h_{1 n}^{*} \mid n \in I\right\}$. Let $r$ be the greatest element in $I$ such that $h_{1 r}^{*} \neq 0$. Using (1) of Lemma $2, h_{j 1}=0$ for $j$ in $J$, hence using (i) $h_{11}^{* \prime}=0$ and therefore $2 \leqq r$. Using (i) we see that the following relations must hold, $h_{1 j}+h_{1 j}^{*}-\sum_{n=2}^{r} h_{1 n}^{*} h_{n j}=0$ for $j=2,3, \ldots, r$ and $h_{1 r}^{*} h_{r, r+1}=0$ (this last result is obtained by using (3) of Lemma 2). At this point we consider the special case when $r=2$. Since $h_{22}=x_{2} f_{22}$ and $h_{12}=k_{1} k_{2}$ we may 
write the first of the above relations as $h_{12}^{*}-h_{12}^{*} x_{2} f_{22}=-k_{1} k_{2}$, this implies by Proposition 1 that $h_{12}^{*}=-k_{1} k_{2}+k_{1} k_{2}\left(x_{2} f_{22}\right)^{\prime}$. Since $0=h_{12}^{*} h_{23}=h_{12}^{*} x_{2} k_{3}$, we obtain $0=-k_{1} k_{2}\left(x_{2}-\left(x_{2} f_{22}\right)^{\prime} x_{2}\right) k_{3}=-k_{1} k_{2} k_{2}^{*} k_{3}$, a contradiction.

We may now assume that $3 \leqq r$. Using properties of $F$ as given in Lemma 2, we rewrite the relations

and $h_{1 r}^{*} h_{r, r+1}=0$ as

$$
h_{1 j}+h_{1 j}^{*}-\sum_{n=2}^{r} h_{1 n}^{*} h_{n j}=0 \text { for } j=2,3, \ldots, r
$$

$$
\begin{gathered}
k_{1} k_{2}+h_{12}^{*}-\sum_{n=2}^{r} h_{1 n}^{*} x_{n} f_{n 2}=0, \\
-h_{1, n-1}^{*} x_{n-1} k_{n}+h_{1 n}^{*}-\sum_{i=n}^{r} h_{1 i}^{*} x_{i} f_{i n}=0 \text { for } n=3,4, \ldots, r, \\
h_{1 r}^{*} x_{r} k_{r+1}=0 .
\end{gathered}
$$

Upon transposition in (iii) we obtain

$$
h_{12}^{*}-h_{12}^{*} x_{2} f_{22}=-k_{1} k_{2}+\sum_{n=3}^{r} h_{1 n}^{*} x_{n} f_{n 2}
$$

We now show by induction on $m$, where $m=2,3, \ldots, r-1$, that (vii) holds.

$$
\begin{aligned}
& h_{1 m}^{*}-h_{1 m}^{*} x_{m}\left(f_{m m}+f_{m m-1} k_{m-1}^{*} k_{m}+f_{m m-2} k_{m-2}^{*} k_{m-1} k_{m-1}^{*} k_{m}+\cdots\right. \\
& \left.+f_{m 2} k_{2}^{*} k_{3} k_{3}^{*} \cdots k_{m-1} k_{m-1}^{*} k_{m}\right) \\
& =-k_{r} k_{2} k_{2}^{*} \cdots k_{m-1} k_{m-1}^{*} k_{m} \\
& +\sum_{i=m+1} h_{1 i}^{*} x_{i}\left(f_{i m}+f_{i m-1} k_{u-1}^{*} k_{m}+f_{i m-2} k_{m-2}^{*} k_{m-1} k_{m-1}^{*} k_{m}+\cdots\right. \\
& \left.+f_{i 2} k_{2}^{*} k_{3} k_{3}^{*} \cdots k_{m-1} k_{m-1}^{*} k_{m}\right) \text {. }
\end{aligned}
$$

When $m=2$ in (vii) we have (vi). Assume that (vii) is valid for $m=p$ where $2 \leqq p$ $<r-1$. Apply Proposition 1 to the inductive hypothesis to solve for $h_{1 p}^{*}$. Substituting this result for $h_{1 p}^{*}$ into (iv) when $n=p+1$, we obtain after collecting terms, simplifying and using the definition of $x_{p}$ the equation (vii) when $m=p+1$.

If we now apply Proposition 1 to (vii) when $m=r-1$, we can solve for $h_{1 r-1}^{*}$. Upon substituting this result for $h_{1 r-1}^{*}$ into (iv) when $n=r$, and upon collecting terms, we obtain

$$
\begin{aligned}
& h_{1 r}^{*}-h_{1 r}^{*} x_{r}\left[f_{r r}+\left(f_{r r-1}+f_{r r-2} k_{r-2}^{*} k_{r-1}+f_{r r-3} k_{r-3}^{*} k_{r-2} k_{r-2}^{*} k_{r-1}\right.\right.+\cdots \\
& \cdot\left(x_{r-1}-\left[x _ { r - 1 } \left(f_{r-1 r-1}+f_{r-1 r-2} k_{r-2}^{*} k_{r-1}\right.\right.\right.\left.+\cdots \quad+f_{r 2} k_{2}^{*} k_{3} k_{3}^{*} \cdots k_{r-1}\right) \\
&\left.\left.\left.\left.+f_{r-1,2} k_{2}^{*} k_{3} k_{3}^{*} \cdots k_{r-1}\right)\right]^{\prime} x_{r-1}\right) k_{r}\right] \\
&=-k_{1} k_{2} k_{2}^{*} \cdots k_{r-1}\left[x_{r-1}-\left[x _ { r - 1 } \left(f_{r-1 r-1}+f_{r-1 r-2} k_{r-2}^{*} k_{r-1}+\cdots\right.\right.\right. \\
&\left.\left.\left.+f_{r-1,2} k_{2}^{*} k_{3} k_{3}^{*} \cdots k_{r-1}\right)\right]^{\prime} x_{r-1}\right] k_{r} .
\end{aligned}
$$

Using the definition of $x_{r-1}$, the last equation may be written as

$$
\begin{gathered}
h_{1 r}^{*}-h_{1 r}^{*} x_{r}\left(f_{r r}+f_{r r-1} k_{r-1}^{*} k_{r}+f_{r r-2} k_{r-2}^{*} k_{r-1} k_{r-1}^{*} k_{r}+\cdots+f_{r 2} k_{2}^{*} k_{3} k_{3}^{*} \cdots k_{r-1} k_{r-1}^{*} k_{r}\right) \\
=-k_{1} k_{2} k_{2}^{*} \cdots k_{r-1} k_{r-1}^{*} k_{r} .
\end{gathered}
$$


Solving this equation for $h_{1 r}^{*}$ by means of Proposition 1 and substituting the result into (v) we obtain

$$
\begin{aligned}
0=-k_{1} k_{2} k_{2}^{*} \cdots k_{r-1} k_{r-1}^{*} k_{r}\left[x_{r}-\left(x _ { r } \left[f_{r r}+f_{r r-1} k_{r-1}^{*} k_{r}\right.\right.\right. & +\cdots \\
& \left.\left.\left.+f_{r 2} k_{2}^{*} k_{3} k_{3}^{*} \cdots k_{r}\right]\right)^{\prime} x_{r}\right] k_{r+1} .
\end{aligned}
$$

Using the definition of $x_{r}$, this last equation may be written as $0=-k_{1} k_{2} k_{2}^{*} \ldots$ $k_{r-1} k_{r-1}^{*} k_{r} k_{r}^{*} k_{r+1}$. This is a contradiction and thus the proof of the theorem is complete.

6. Remarks. A ring $R$ is said to be right vanishing if, given any sequence $\left\{a_{n} \mid n=1,2, \ldots\right\}$ of elements of $R$, there exists a positive integer $r$, depending on the sequence, such that $a_{1} a_{2} \cdots a_{r}=0$. As stated in the introduction, Patterson [3] has proved: $\Gamma\left(R_{J}\right)=(\Gamma(R))_{J}$ if and only if $\Gamma(R)$ is right vanishing. This result is also a consequence of the theorem we have proved.

When $\Gamma(R)$ is a prime ring the theorem gives a simple characterization of $\Gamma\left(R_{J}\right)$. In this case $\Gamma\left(R_{J}^{\prime}\right)$ coincides with the set of matrices in $(\Gamma(R))_{J}$ which have the property that all but a finite number of columns are zero. For if $A$ is such a matrix, then $\left\{\mathfrak{A}_{\lambda} \mid \lambda \in J\right\}$ is a right vanishing family of left ideals of $R$ and therefore, by the theorem, $A$ is in $\Gamma\left(R_{J}\right)$. Conversely, if $A$ is in $\Gamma\left(R_{J}\right) \leqq(\Gamma(R))_{J}$ and if $A$ has an infinite number of columns which contain nonzero elements, then there are distinct indices $\lambda_{1}, \lambda_{2}, \ldots$ such that $\mathfrak{A}_{\lambda_{n}} \neq 0$. Then, since $\Gamma(R)$ is a prime ring, we can show, by induction, that for each $r$ there exist $a_{n} \in \mathfrak{A}_{\lambda_{n}}(n=1,2, \ldots, r)$ such that $a_{1} a_{2} \ldots$ $a_{r} \neq 0$. For if $0 \neq b$ is in $\mathfrak{A}_{\lambda_{r+1}}$ then $0 \neq a_{1} \cdots a_{r} \Gamma(R) b \leqq a_{1} \cdots a_{r} \mathfrak{A}_{\lambda_{r+1}}$, hence there exists $a_{r+1}$ in $\mathfrak{A}_{\lambda_{r+1}}$ such that $a_{1} \cdots a_{r} a_{r+1} \neq 0$. This contradicts the fact, given by the theorem, that $\left\{\mathfrak{A}_{\lambda} \mid \lambda \in J\right\}$ is a right vanishing family of left ideals of $R$.

The authors would like to thank Professor S. A. Amitsur for his helpful suggestions and discussions regarding the sufficiency half of the proof of the theorem. The characterization when $\Gamma(R)$ is a prime ring was also pointed out by Professor Amitsur and extended the same result obtained by the authors previously in the case when $\Gamma(R)$ is a nonzero integral domain.

\section{REFERENCES}

1. N. Jacobson, Structure of rings, Amer. Math. Soc. Colloq. Publ. Vol. 37, Amer. Math. Soc., Providence, R. I., 1956.

2. E. M. Patterson, On the radical of certain rings of infinite matrices, Proc. Roy. Soc. Edinburgh Sect. A 65 (1957/1961), 263-271.

3. - - On the radicals of rings of row-finite matrices, Proc. Roy. Soc. Edinburgh Sect. A 66 (1961/1962), 42-46.

UNIVERSITY OF UTAH, Salt Lake City, Utah

UNIVERSITY OF ILLINOIS,

URBANA, ILLINOIS 